\title{
CAMBIOS CLIMÁTICOS Y ESTRATEGIAS TECNOLÓGICAS: CASO ARQUEOLÓGICO ARROYO MALO 3, SUR DE MENDOZA, ARGENTINA
}

\author{
Mudanças climáticas e estratégias tecnológicas: \\ Caso arqueológico Arroyo Malo 3, sul de Mendoza, Argentina
}

Cecilia Pérez Winter

Universidade de Buenos Aires (UBA)

Buenos Aires - Argentina

cecipw@gmail.com

Artigo recebido para publicação em 14/08/2009 e aceito para publicação em 05/11/2009

RESUMEN: Hoy en día la comunidad científica y la sociedad están preocupados con los cambios climáticos que están aconteciendo desde años. La realidad es que cambios climáticos existieron a lo largo de la historia de nuestro planeta. La diferencia que se plantea con los cambios más recientes, es que el humano es en gran parte responsable. Más allá de esto, los cambios climáticos suelen producir cambios en la forma en que nosotros hacemos las cosas. Es decir, en el cómo enfrentar ciertos cambios naturales, como ser una sequía. En este trabajo se presenta un caso arqueológico, sobre las posibles estrategias que pudieron ser generadas por los cazadores recolectores que ocuparon el sur de Mendoza hace 5.000 años, para lidiar con un proceso de aridización ocurrido durante el Holoceno medio (8.000 - 4.000 años atrás). Se propone que los grupos humanos pudieron haber cambiado su forma de organizar su tecnología lítica para enfrentar el impacto del proceso de aridez.

Palabras claves: Estrategias humanas, Tecnología lítica, Holoceno medio

RESUMO: Hoje a comunidade científica e a sociedade estão preocupadas com as mudanças climáticas que estão ocorrendo nos últimos anos. Mas a realidade é que estas mudanças sempre existiram ao longo da historia da terra. A diferença é que, nas últimas mudanças, o humano é o responsável por grande parte delas. Além disso, as mudanças climáticas geram mudanças na forma de fazermos as coisas. Ou seja, em como enfrentar os problemas gerados por essas mudanças naturais, por exemplo, uma seca. Neste artigo está apresentado um caso arqueológico, sobre as possíveis estratégias que provavelmente tenham sido feitas por caçadores - coletores que habitaram o sul de Mendoza há 5.000 anos, para lidar com processos de aridez ocorridos durante o Holoceno médio (8.000 - 4.000 anos atrás). Propõe- se que os grupos humanos poderiam ter trocado a forma de organizar a sua tecnologia litica para enfrentar o impacto do processo de aridez.

Palavras-chave: estrategias humanas, tecnologia litica, Holoceno médio 


\section{INTRODUCCIÓN}

El humano se desarrolla y desarrolló en un contexto ambiental que es variable en tiempo y espacio (CASHDAN, 1992). Los cambios climáticos significativos producen cambios culturales (BRYSON, 1994) debido a las respuestas sociales y culturales que generan los grupos humanos para amortiguar y resolver los problemas que el ambiente impone (HALSTEAD y O’SHEA, 1989). Algunos cambios climáticos que ocurren a nivel global, impactan con distinta intensidad y duración según cada ambiente local (GAMBLE y SOFFER, 1990; JONES et al. 1999; MARKGRAF, 1983). Es en relación a esto que muchos investigadores recalcan la importancia de integrar los datos paleoambientales con los del registro arqueológico (BRYSON, 1994; DINCAUZE, 2000; GAMBLE y SOFFER, 1997; JONES et al. 1999). Ya que esto permite entender una parte del comportamiento y de las estrategias que desarrollaron los grupos humanos a lo largo del tiempo (HASTLED y O'SHEA, 1989; JONES et al. 1999; KELLY, 1995; MELTZER, 1999; YACOBACCIO, 1996; WINTERHALDER y GOLAND, 1997, entre muchos otros).

En el caso de las condiciones climáticas ambientales que se dieron en el transcurso del Holoceno medio, el proceso de aridización y la coincidente falta de registro arqueológico, son dos características que se producen en distintas partes de América (ARAUJO et al.2005; BRACCO et al. 2005; MELTZER, 1999; NUÑEZ et al. 2002; YACOBACCIO y MORALES, 2005). En el sur de Mendoza, esta falta de registro arqueológico durante el Holoceno medio va desde los 7.000 a 5.300 años atrás (PÉREZ WINTER, 2009a). Según Gil et al. (2005, p. 89), el proceso de aridización pudo haber reducido la biomasa y las fuentes de agua que estaban disponibles, provocando cambios en la intensidad de ocupación, uso del espacio, relaciones sociales, movilidad y demografía de los grupos humanos que ocuparon la región. Este proceso pudo provocar que los recursos necesarios para la subsistencia no estén igualmente disponibles y que los grupos humanos hayan tenido que generar estrategias para obtener dichos recursos.

Si la aridez característica del Holoceno medio (8.000 - 4.000 años) produjo estrés en los recursos de subsistencia (como el guanaco y otras especies vegetales), esto pudo generar cambios en la organización y las estrategias implementadas por los grupos humanos en el pasado para la adquisición de estos recursos. Es en este sentido, que este trabajo plantea algunas estrategias tecnológicas que pudieron ser utilizadas por los antiguos ocupantes del sur de Mendoza, Argentina, para enfrentar los problemas que pudo producir el proceso de aridización ocurrido durante el Holoceno medio. El área de estudio es el alto valle del río Atuel (AVRA), y se contrastarán algunas hipótesis de trabajo a través del análisis del material lítico del sitio Arroyo Malo 3 (AMA 3).

\section{2. ÁREA Y CASO DE ESTUDIO}

El AVRA se localiza en la Cordillera Andina Meridional dentro de la Cordillera Principal, la cual incluye toda la cuenca cordillerana del Atuel (GONZÁLEZ DÍAZ y FAUQUÉ, 1993). Está limitada por la cuenca del río Diamante al norte, la del río Salado al sur; la divisoria de aguas argentino-chilena al oeste y el comienzo del piedemonte andino al este (FIG. 1). El área se ubica entre los $34^{\circ} 30^{\prime}$ y $35^{\circ} 10^{\prime}$ de LS y los $70^{\circ}$ y $69^{\circ} 30^{\prime}$ de LO. Tiene unos $90 \mathrm{~km}$ en el eje Este-Oeste por $20 \mathrm{~km}$ en el eje Norte-Sur, encerrando un gradiente altitudinal que va desde los 1.500 m.s.n.m en el piedemonte, hasta alturas de más de 5.000 m.s.n.m en el cordón del límite. A medida que se aumenta en altitud, disminuye la temperatura en $0,6^{\circ} \mathrm{C} /$ $100 \mathrm{~m}$ en verano y $4^{\circ} \mathrm{C} / 100 \mathrm{~m}$ en invierno (NORTE, 2000, p. 27; PROHASKA, 1976). La biomasa primaria también disminuye, cerca de -0,5 NDVI-I a más de 2.000 m.s.n.m., según el índice de vegetación normalizada propuesta por Paruelo et al. (2001).

El sitio arqueológico AMA 3 es un alero de andesita perteneciente a la Formación Cerro Guanaquero (VOLKHEIMER, 1978), ubicado a los $34^{\circ} 52^{\prime} 22^{\prime \prime}$ LS y $69^{\circ} 54^{\prime} 14^{\prime \prime}$ LO a 2.000 m.s.n.m., sobre la margen derecha de arroyo Malo, tributario del río Atuel (FIG. 1). El alero tiene $13 \mathrm{~m}$ de abertura, 7,5 $\mathrm{m}$ de profundidad y $3 \mathrm{~m}$ de altura. Está orientado con una dirección aproximada oeste-este y la superficie excavada del mismo fue de $6 \mathrm{~m}^{2}$. Tiene una secuencia de ocupación que va desde ca. 8.900 años AP hasta momentos históricos (DIEGUEZ y NEME, 2003). El 


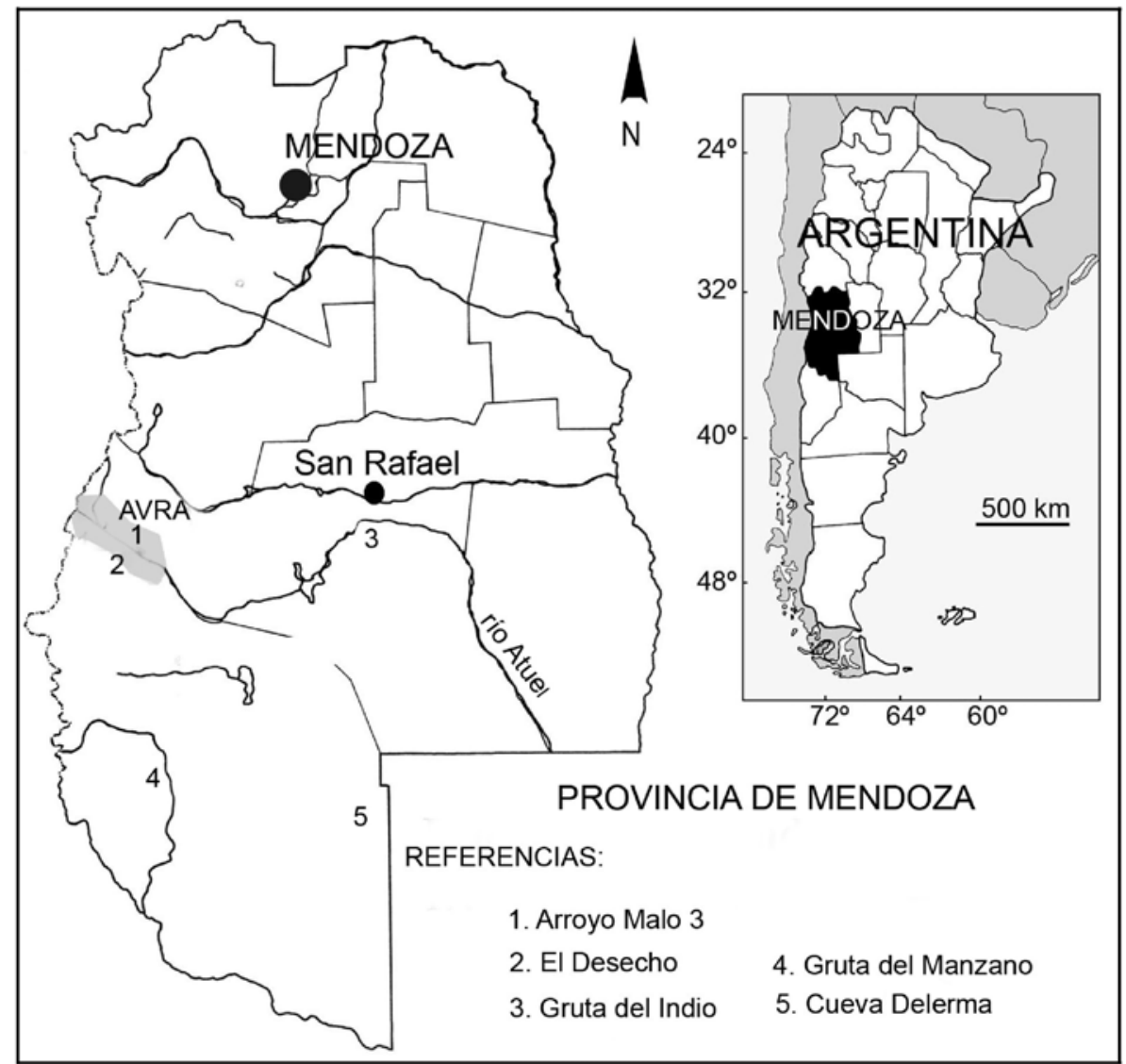

FIGURA 1: Localización del área de estudio y de los sitios arqueológicos correspondientes al Holoceno medio.

paisaje en el cual está emplazado, presenta morenas de la última glaciación, terrazas, depósitos coaluviales, eólicos y aluviales. También se encuentran coladas basálticas provenientes del cerro El Sosneado, que recorre al este todo el lugar con una dirección aproximada de norte - sur, conformando una gran cantidad de reparos de variadas dimensiones (VOLKHEIMER, 1978).

Las evidencias recolectadas del sitio AMA 3 muestra que las actividades allí realizadas fueron limitadas (como talla y reacondicionamiento de instrumentos, y el procesamiento de vertebrados). Sin embargo, a partir de los 2.200 años atrás, las actividades realizadas en el sitio se habrían ampliado al incorporarse el procesamiento de vegetales, con una mayor recurrencia de ocupación en el lugar (LLANO, 2005). La funcionalidad del sitio, como estación de caza, parece haberse mantenido entre los 8.700 y los 2.200 años atrás (véase PÉREZ WINTER, 2009a)

\subsection{Registro Paleoambiental}

En una escala continental, los registros de Sudamérica indican aridez y temperaturas en ascenso para el Holoceno medio (ARAUJO et al. 2005; NUÑEZ et al. 2002; MANICINI et al. 2005). Según estudios recientes (ABRAHAM et al. 2000, p. 60), el debilitamiento de la presión y la temperatura provocaron una disminución en el intercambio de las masas de aires, que generó una fase de aridización. Sin embargo, esto varía según el tipo de ambiente en sentido longitudinal y latitudinal (ZÁRATE, 2002). En el sur de Mendoza, entre los 8.500 y 5.000 años AP, en el piedemonte oriental (660 m.s.n.m.), un aumento en la temperatura desarrolló un desierto arbustivo postglaciar, surgiendo un decrecimiento de la precipitación veraniega, lo que dio paso a una vegetación de tipo Monte (D'ANTONI, 1980). Este momento parece haber estado caracterizado por condiciones de extrema aridez con tempera- 
turas más altas que las que hoy presenta la región (MARKGRAF, 1983). En el caso del río Atuel, hacia los 5.000 y 4.500 años atrás existe una etapa de activa agradación. Las facies de grava basales con terrazas de 8 a $10 \mathrm{~m}$ sugieren condiciones de alta energía y caudales importantes (ZÁRATE, 2002, p. 37). Esto concordaría con lo propuesto desde el análisis polínico realizado por D'Antoni (1983), en un aumento generalizado de la temperatura que afectó tanto el piedemonte y llanura como a la Cordillera, con el derretimiento de los glaciares y el incremento del caudal de agua. Sin embargo, Markgraf (1983), tomando evidencia polínica, interpreta una disminución en la temperatura en la alta montaña junto a un incremento del caudal del Atuel y ascenso de los niveles freáticos.

Entre los 8.000 y 4.000 años atrás, el límite de la región árida - semiárida, fue desplazado hacia el este (MANCINI et al. 2005). Su límite oeste alcanzó el máximo desplazamiento hacia el este en el extremo sur de la Patagonia hace 6.000 años atrás. Al mismo tiempo, en el centro de la región árida - semiárida, se desarrollaron arbustos de estepa y vegetación de Monte, sugiriendo un máximo de condiciones secas y el establecimiento de la Diagonal Árida Sudamericana (DAS) al norte de los $42^{\circ} \mathrm{S}$. Hace 6.000 años atrás, la DAS, aumentó su longitud, extendiéndose desde los $42^{\circ}$ hasta los $32^{\circ} \mathrm{S}$.

En las zonas de altura (2.000 m.s.n.m.), los estudios de microvertebrados en AMA 3 (8.000 años atrás), coincide con un aumento de temperatura por la presencia de especies de ambientes más cálidos (NEME et al. 2002). Stingl y Garlef (1985) mencionan que para el AVRA entre los 6.000 y 4.000 años atrás, se produjeron avances glaciarios como consecuencia de un aumento en las precipitaciones.

En síntesis, los registros glaciológicos, aluviales y de polen están mostrando condiciones más áridas durante el Holoceno medio. Pasando de condiciones frías y de mayor disponibilidad de agua en cuerpos lagunares y cursos de aguas, a condiciones áridas y de mayor temperatura (ZÁRATE, 2002). La causa de esta mayor aridez generalizada durante el Holoceno medio, tanto en las zonas bajas como las altas del sur mendocino, se atribuiría a una mayor influencia de los vientos del oeste, producto del desplazamiento hacia el norte de los centros anticiclónicos del pacífico. Sin embargo, esto produjo condiciones ambientales diferentes. Por un lado, el aumento en las precipitaciones de invierno de alta Cordillera. Por el otro, una disminución de las lluvias de verano en las zonas bajas, por una menor influencia del anticiclón del Atlántico (ZÁRATE, 2002; GIL et al. 2005).

\subsection{Registro Arqueológico del Sur de Mendoza}

Durante el periodo correspondiente al Holoceno medio, en el sur de Mendoza, solo se tiene registro de cinco sitios arqueológicos: Gruta del Manzano (FIG. 1) con un fechado de $c a$. 7.200 años AP, Gruta del Indio (FIG. 1) con un fechado de entre 7.800 y 7.400 años atrás; cueva Delerma con un fechado de ca. 7.600 años atrás; AMA 3 (FIG. 1) con fechados de ca. 7.600 y 5.300 años atrás, y Arroyo el Desecho (FIG. 1) con un fechado de 5.500 atrás.

En el valle del río Grande, la Gruta del Manzano es el único sitio con evidencia de ocupación durante el primer milenio del Holoceno medio. En base a las evidencias líticas (puntas apenduculadas, micro raspadores) y por su semejanzas de conjunto y cronología con otro sitio de Neuquén, Durán (2004), caracteriza esa ocupación dentro de un proceso de colonización. Para estos momentos se estaría utilizando el sitio dentro de un circuito de explotación plurianual (DURÁN, 2004, p. 90). Después de los 7.200 y hasta los 3.800 años atrás. Durán registró un evento volcánico que explicaría la ausencia de ocupación humana en el área (DURÁN, 2004, p. 90).

En el Atuel medio, fuera del sector cordillerano, la evidencia de ocupación proviene del sitio Gruta del Indio. Para Lagiglia (2002), antes de los 3.000 años atrás, se estaba produciendo un cambio hacia la producción de alimentos, proceso que parece interrumpirse por un hiatus de casi 3.000 años. Este hiatus va desde los 7.430 hasta los 3.800 años atrás. La ocupación en el sitio se retoma a los 3.800 años, con características distintas (LAGILIGA 1997a y b, 2002). Lagiglia (1997a, 2002) planteó que durante el Holoceno medio hubo un aumento en la movilidad de los grupos, justificando de esta manera el escaso registro arqueológico correspondiente al Holoceno medio (LAGIGLIA 2001).

En el área de La Payunia, en el límite con La 
Pampa y lejos de las vertientes de agua (2 km), se ubica Cueva Delerma que tiene un fechado de ca. 7.600 años AP. La evidencia de su ocupación fue interpretada como efímera, dado que sólo se encontró unas decenas de lascas, un fragmento distal de punta y especímenes óseos (GIL, 2006, p.107). Gil (2006, p. 107) propone que el sitio fue utilizado en la exploración de nuevas áreas y/o en forma efímera para determinados trayectos en el territorio, funcionando como un campamento de viajeros. Luego, cerca de los 5.000 años, se produce un hiatus ocupacional. El reinicio de las ocupaciones se registra con el sitio La Corredera, en ca. 2.000 años AP, donde Gil (2006, p. 121), propone una exploración o colonización del área.

En el AVRA el único sitio con evidencia de ocupación durante el Holoceno medio es AMA 3. Las tendencias en la evidencia lítica sugieren que durante ese período se implementaron estrategias de equipamiento personal y conservada, característica de grupos con alta movilidad, además de una corta duración en la ocupación del sitio. Asimismo, durante ese periodo parece que se estaba produciendo una reexploración del área (PÉREZ WINTER, 2009a).

En el valle del Salado, se encuentra localizado el sitio Arroyo "El Desecho". Este sitio tiene un fechado similar a AMA 3 y fue excavado por Lagiglia en 1999. De esas excavaciones se recuperaron puntas pedunculadas de tipo andina confeccionados en basalto y obsidiana, y un entierro secundario que arrojó un fechado de ca. 5.500 años (LAGIGLIA, 2005).

Algunas explicaciones fueron propuestas para explicar el registro arqueológico correspondiente al Holoceno medio. Durán (2004) plantea que el hiatus registrado en el área de río Grande es una consecuencia de los eventos volcánicos ocurridos durante el Holoceno medio. Por su parte, Lagiglia (2001) propone que es reflejo de un aumento en la movilidad, mientras que Garvey (2008) atribuye esta falta de datos en el sur de Mendoza a tres cuestiones que no son excluyentes: pobre visibilidad arqueológica, baja densidad poblacional y al comportamiento adaptativo humano. Asimismo, esta autora propone que la movilidad residencial de los grupos debería disminuir al escasear los recursos.

Sin embargo, otros investigadores plantean que el registro del Holoceno medio estaría reflejando cambios culturales inducidos por condiciones de estrés ambiental, debido a la aridez que imperó entre los 6.000 y 4.000 años atrás. Dicha aridización pudo haber reducido la biomasa y las fuentes de aguas que estaban disponibles en el ambiente. Cabe destacar que el sur de Mendoza es un ambiente árido-semiárido, y este cambio climático pudo influir en la intensidad de ocupación, el uso del espacio, las relaciones sociales, la movilidad y demografía de los grupos humanos que ocuparon la región (GIL et al. 2005, p. 89).

\subsection{Cambios Ambientales y Estrategias Tecnológicas}

Los cambios climáticos pueden provocar cambios en la organización de los grupos. Sin embargo, no hay que dejar de tener en cuenta que éstos cambios son percibidos por los grupos humanos, que también generan cambios en su entorno, especialmente a través de su tecnología (DINCAUZE, 2000). Esta última permite la resolución de los impedimentos que el ambiente impone (TORRENCE, 1989). Nelson (1991, p. 60) afirma que: "Humans are viewed as decision makers within a variable environment; ecological structure is viewed as conditioning behavior to some degree. Optimal or suitable choices among alternatives can be understood only within context of environmental conditions and available technological capabilities.”

Es en este sentido que, la tecnología lítica se desarrolla para resolver problemas y los instrumentos son utilizados por los individuos como parte de estrategias para lidiar con su ambiente social y ambiental (NELSON, 1991; TORRENCE, 1989). Además, a través de ella también se pueden generar respuestas para disminuir o evitar el riesgo ante la escasez de recursos, por ser una de las formas en la que los grupos humanos se organizaron para acceder a los recursos de subsistencia (BAMFORTH y BLEED, 1997; BOUSMAN, 1993, 2005; FRANCO, 2004; TORRENCE, 1989; WIESNNER, 1982).

El proceso de aridización que caracterizó al Holoceno medio, pudo provocar un estrés en los recursos. El estrés puede ocurrir cuando aumenta la población, declina la productividad del ambiente o por cambios climáticos. Los mecanismos que propone Nelson (1995) para resolver esta situación son: 
especialización y diversificación. El primero, se refiere a focalizarse en uno o pocos recursos, convirtiéndose esos pocos recursos en el foco de la subsistencia. Los instrumentos, por lo tanto, deben ser eficientes para adquirir y procesar los recursos, y obtener la mayor cantidad posible. Un diseño confiable es importante para minimizar la posibilidad de pérdida (NELSON, 1995, p. 114). Sin embargo, los instrumentos variarán según los recursos que se quieran obtener, por ejemplo, en el caso de la caza, las armas deben ser eficientes, confiables y estandarizadas (NELSON, 1995, p. 118).

La diversificación, puede adoptar dos formas: a) los grupos se focalizan en pocos recursos, diversificando sus técnicas de obtención de recursos; o b) los grupos diversifican los recursos que habrán de consumir. Se requiere más tiempo en la búsqueda de alimentos que en una estrategia de especialización. Por lo tanto, los instrumentos con diseños versátiles, flexibles y transportables serán priorizados (NELSON, 1995, p. 121). Si la estrategia es la caza, seguramente el arco y la flecha serán más pequeños que los utilizados por los cazadores especializados. Una estrategia de diversificación, entonces, combinará la versatilidad, flexibilidad, portabilidad y confiabilidad. De esta forma, en tiempos de estrés, la organización de la tecnología y el diseño de los instrumentos responderán de manera óptima hacia los problemas de subsistencia (NELSON, 1995, p. 135). En el caso del sur de Mendoza, el estrés de recursos pudo provocar que la tasa de encuentro de guanaco disminuyera. Sin embargo continúo siendo el animal más consumido durante el Holoceno medio (GARVEY, 2008). A partir de los 2.000 años es que se registra una amplitud en la dieta, registrándose semillas quemadas y animales de menor porte (NEME, 2007). Por lo tanto se puede decir, que desde la subsistencia hubo una estrategia de especialización durante el Holoceno medio.

Asimismo, la tecnología cumple un rol importante en determinar las elecciones económicas de los grupos humanos en aspectos como disminución de riesgo. En el caso de Bousman (1993), él diferencia instrumentos de mantenimiento e instrumentos extractivos y la estrategia que se aplica en cada uno (conservación y expeditividad). La estrategia de conservación es aquella en la que el instrumento producido es cuidadosamente conservado, reforma- tizado, almacenado, transportado y está en directa relación con su manufactura anticipada a la actividad a realizar (BINFORD, 1979). Esta estrategia mitiga la incongruencia entre la disponibilidad de instrumentos o materia prima y la ubicación en donde se realizan y utilizan los instrumentos. También resuelve el problema de adquirir recursos móviles y de la falta de materia prima o instrumentos, asegurando el tenerlos cuando se los necesita (CARR, 1994; NELSON, 1991: 63). La estrategia expeditiva, en contraposición, serían aquellos instrumentos manufacturados con una menor inversión de trabajo y descartados en su inmediato lugar de uso (BINFORD, 1973:242). Esta estrategia anticipa la presencia de suficientes materiales y tiempo. Depende de tres condiciones: a) que la materia prima esté cerca del lugar donde se lleven a cabo las actividades; b) disponibilidad de tiempo para manufacturar los instrumentos como parte de la actividad; c) larga ocupación o uso regular (NELSON, 1991: 64). Esta estrategia sería minimizadora de esfuerzo tecnológico, manufacturando los instrumentos en el lugar que serán utilizados (CARR, 1994: 36)

En cuanto a los instrumentos extractivos que propone Bousman (1993), estos son los utilizados para obtener los recursos alimenticios; y los de mantenimiento, para manufacturar o reparar otros implementos tecnológicos, como los instrumentos extractivos. El tipo de estrategia en cada tipo de instrumento se puede observar en la frecuencia y el estado de descarte del instrumento. En una estrategia recolectora, donde los recursos son menos predecibles y abundantes, se espera que haya más mantenimiento y vida útil en los instrumentos de mantenimiento, y diseños confiables y descartados con mayor frecuencia en los instrumentos extractivos. En contraposición, se espera lo contrario en una estrategia forrajeadora, donde los recursos son más abundantes y con mayor disponibilidad.

En otro artículo más reciente, Bousman (2005) ejemplifica esta propuesta con un caso arqueológico proveniente del alero Blydefontein, con evidencia de ocupación correspondiente a la Edad de Piedra Tardía en el sur de África. En este análisis intenta integrar los conceptos desarrollados por el forrajeamiento óptimo y la teoría de la organización tecnológica. Supone que el tiempo y la severidad de las sequías registradas son las dos fuerzas "selectivas" que influyen en la tecnología 
de los cazadores recolectores. Propone que los cazadores recolectores de la Edad de Piedra Tardía diseñaron y utilizaron su tecnología, por lo que fueron sensibles a la fluctuante disponibilidad de los recursos.

Diferencia dos estrategias que deberían ser utilizadas en diferentes condiciones de riesgo, los minimizadores de tiempo y los maximizadores de recursos. La primera estrategia correspondería a situaciones de menor riesgo y constaría de un uso conservado de los instrumentos extractivos y un uso expeditivo de los instrumentos de mantenimiento. En cambio, los maximizadores de recursos, debería ocurrir en condiciones de mayor riesgo, con un uso conservado de los instrumentos de mantenimiento y un uso expeditivo de los instrumentos extractivos. Para medir la severidad del riesgo toma datos paleoambientales $\mathrm{y}$, junto a las evidencias arqueológicas, diferencia dos patrones: 1) un momento húmedo de menor riesgo, donde se consumieron animales de gran porte, con menor reformatización de raspadores, materia prima local y pocos microlitos enteros; 2) un momento de condiciones más secas, evidencia de consumo de animales de menor porte y mayor evidencia de reformatización en los raspadores, asimismo se observó un aumento en las materias primas no locales y más cantidad de microlitos enteros descartados. El primer escenario respondería a una estrategia de minimizador de tiempo, mientras que el segundo a la de un maximizador de recursos.

\subsection{Hipótesis de trabajo}

Partiendo de los modelos de organización de tiempo, algunos investigadores postulan que los cazadores recolectores gastan su tiempo en varias actividades (BOUSMAN, 1993; UGAN et al. 2003). Estas pueden ser: búsqueda de pareja y cuidado de la descendencia, obtención de comida, abrigo, diseño y mantenimiento de instrumentos, relajación, actividad ritual y socialización (BOUSMAN, 1993, p. 63). La disponibilidad de tiempo es limitada y cada actividad tiene costos y beneficios diferenciales para la adaptabilidad del individuo y el grupo, por lo tanto, estas actividades son mutuamente excluyentes (BOUSMAN, 1993). La cantidad de tiempo y energía disponible por un grupo particular puede verse reflejada en el tipo de materia prima utilizada, como así también, la energía invertida en la manufactura de instrumentos. Asimismo, la falta de energía puesta en el aprovisionamiento de materia prima puede ser por invertir mayor energía en otro tipo de actividades socialmente adaptativas (JESKE, 1992, p. 467).

Tomando como partida que durante el Holoceno medio hubo un estrés de recursos, y teniendo en consideración lo anteriormente planteado sobre la disponibilidad del tiempo en los grupos cazadores recolectores, se proponen dos hipótesis sobre las posibles estrategias en la tecnología lítica que pudieron ser implementadas por los grupos que ocuparon el AVRA:

1. "Durante el Holoceno medio los grupos utilizaron una estrategia de maximización en la explotación de recursos.” En esta estrategia se espera un uso conservado de los instrumentos de mantenimiento y un uso expeditivo de los instrumentos extractivos. Además, se espera una mayor frecuencia de instrumentos extractivos manufacturados en materias primas de muy buena y buena calidad para la talla (sea local o foránea), en su mayoría enteros y activos. Para los instrumentos de mantenimiento se espera una menor frecuencia, que estén agotados, fracturados y manufacturados en materia prima local o de menor calidad que los instrumentos extractivos.

Sin embargo, también se puede plantear una hipótesis alternativa:

2. "Durante el Holoceno medio se dedicó más tiempo y energía a las actividades de subsistencia, dedicando menos tiempo y energía a las actividades tecnológicas.” En este caso se espera que el conjunto lítico esté representado por instrumentos confeccionados con una menor inversión de energía, predominio de instrumentos manufacturados en materia prima local, y la utilización de técnicas de reducción menos costosas, como la reducción unifacial por sobre la bifacial.

\section{RESULTADOS DEL ANÁLISIS}

La muestra del material lítico correspondiente al sitio AMA 3, tiene un total de 2.632 piezas líticas, de los cuales, 119 son chunks, que son aquellos fragmen- 
tos poliédricos que tienen 3 o más caras en las que no se pueden reconocer las bocas de lascado y no fueron considerados en el análisis. También se registraron 938 desechos fracturados sin talón, 1.225 desechos enteros y fracturados con talón, 13 instrumentos, 13 manos/ molinos, 12 núcleos y 3 percutores (véase TAB. 1). En este trabajo se hará hincapié en la comparación de los instrumentos y su relación con las materias primas utilizadas, para contrastar las hipótesis propuestas.

TABELA 1: Materiales líticos correspondientes al sitio AMA 3.

\begin{tabular}{ccccccc}
\hline Conjuntos & Desechos & Instrumentos & Manos/molinos & Núcleos & Percutores & Total general \\
2 & $494(96,30 \%)$ & $7(1,36 \%)$ & $8(1,56 \%)$ & $3(0,58 \%)$ & $1(0,19 \%)$ & 513 \\
3 & $312(97,52 \%)$ & $3(0,93 \%)$ & $1(0,31 \%)$ & $3(0,93 \%)$ & $1(0,31 \%)$ & 320 \\
4 & $417(96,75 \%)$ & $3(0,70 \%)$ & $4(0,93 \%)$ & $6(1,39 \%)$ & $1(0,23 \%)$ & 431 \\
\hline
\end{tabular}

Asimismo, la secuencia de ocupación del sitio AMA 3, fue dividida en tres conjuntos temporales que se puede observar su lapso temporal, duración y correspondencia con las etapas del Holoceno, en la TAB. 2.

TABELA2: La secuencia de ocupación está dividida en tres conjuntos temporales:

\begin{tabular}{cccc}
\hline Conjuntos & Lapso & Duración temporal & Holoceno \\
Conjunto 4 & $7600-8900$ años atrás & 1.355 años & temprano \\
Conjunto 3 & $5000-7600$ años atrás & 2.360 años & medio \\
Conjunto 2 & $2200-5000$ años atrás & 3.060 años & tardío \\
\hline
\end{tabular}

\subsection{Materias primas}

Las materias primas fueron determinadas macroscópicamente y a partir de su textura se caracterizó nominalmente sus calidades para la talla en: muy buenas, buenas y regular (PÉREZ WINTER, 2009a). Siguiendo estos criterios, la obsidiana (Obs.) y el sílice (Sil.) son de muy buena calidad, el basalto 1 (Bas. 1) y 3 (Bas. 3) son de buena calidad y el basalto tipo 2 (Bas. 2) es de calidad regular. Los basaltos se encuentran en los alrededores del sitio (PÉREZ WINTER, 2009b), sin embargo, estos tres tipos no fueron registrados en sus cercanías, hasta el momento (PÉREZ WINTER, 2009a).

En la tabla 3, se puede observar que la materia prima más representada para la manufactura de instrumentos es Bas. 1. En general, los instrumentos están manufacturados en materias primas de muy buena y buena calidad para la talla.

\subsection{Instrumentos}

La TAB. 3 muestra los porcentajes y frecuencias de los instrumentos registrados en los tres conjuntos temporales. El conjunto 3 (correspondiente a la ocupación del Holoceno medio) es el que menos instrumentos tiene. Estos son una preforma de bas. 1, un fragmento de filo de bas. 2 y una muesca lateral de sílice (FIG. 2). El primero y el último están confeccionados en materias primas de buena y muy buena calidad. El conjunto 2 (Holoceno tardío) es el que mayor cantidad y variabilidad de instrumentos tiene, formatizados todos en materias primas de buena y muy buena calidad. Este conjunto parece tener una mayor inversión de energía puesto en la confección de los instrumentos, en comparación a los conjuntos 3 y 4 , ya que el conjunto 2 , tiene un raspador junto con un filo, fragmentos de puntas de proyectil. El conjunto 4 (Holoceno temprano), tiene la misma cantidad de instrumentos que el conjunto 3, de los cuales dos de ellos están fracturados. 
TABELA 3: Tipos de instrumentos registrados en cada conjunto.

\begin{tabular}{|c|c|c|c|c|c|c|c|c|c|c|c|c|}
\hline & \multicolumn{12}{|c|}{ INSTRUMENTOS } \\
\hline & \multicolumn{4}{|c|}{$\overrightarrow{\dot{m}}$} & $\stackrel{\sim}{\dot{\oplus}}$ & \multicolumn{2}{|c|}{$\stackrel{m}{\dot{\theta}}$} & \multicolumn{2}{|c|}{$\dot{\Xi}$} & $\dot{\hat{O}}$ & \multicolumn{2}{|c|}{$\dot{\bar{n}}$} \\
\hline & 焉 & 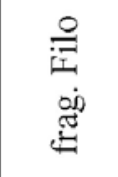 & 总芯 & 焉总 & 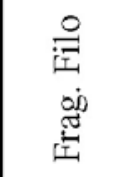 & 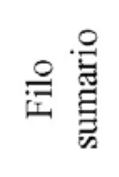 & 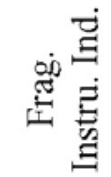 & 畄 & 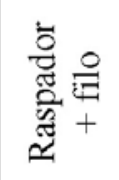 & 焉怘 & 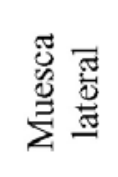 & $\stackrel{+}{\stackrel{\Xi}{\Xi}} \underset{\Xi}{\Xi}$ \\
\hline 2 & $\begin{array}{c}1 \\
(14,29 \\
\%) \\
\end{array}$ & - & $\begin{array}{c}1 \\
(14,29 \\
\%) \\
\end{array}$ & - & - & $\begin{array}{c}1 \\
(14,29 \\
\%) \\
\end{array}$ & $\begin{array}{c}1 \\
(14,29 \\
\%) \\
\end{array}$ & - & $\begin{array}{c}1 \\
(14,29 \\
\%) \\
\end{array}$ & $\begin{array}{c}1 \\
(14,29 \\
\%) \\
\end{array}$ & - & $\begin{array}{c}1 \\
(14,29 \\
\%) \\
\end{array}$ \\
\hline 3 & - & - & - & $\begin{array}{c}1 \\
(33,33 \\
\%) \\
\end{array}$ & $\begin{array}{c}1 \\
(33,33 \\
\%) \\
\end{array}$ & - & - & - & - & - & $\begin{array}{c}1 \\
(33,33 \\
\%) \\
\end{array}$ & - \\
\hline 4 & - & $\begin{array}{c}1 \\
(33,33 \\
\%) \\
\end{array}$ & - & - & $\begin{array}{c}1 \\
(33,33 \\
\%)\end{array}$ & - & - & $\begin{array}{c}1 \\
(33,33 \\
\%)\end{array}$ & - & - & - & - \\
\hline
\end{tabular}

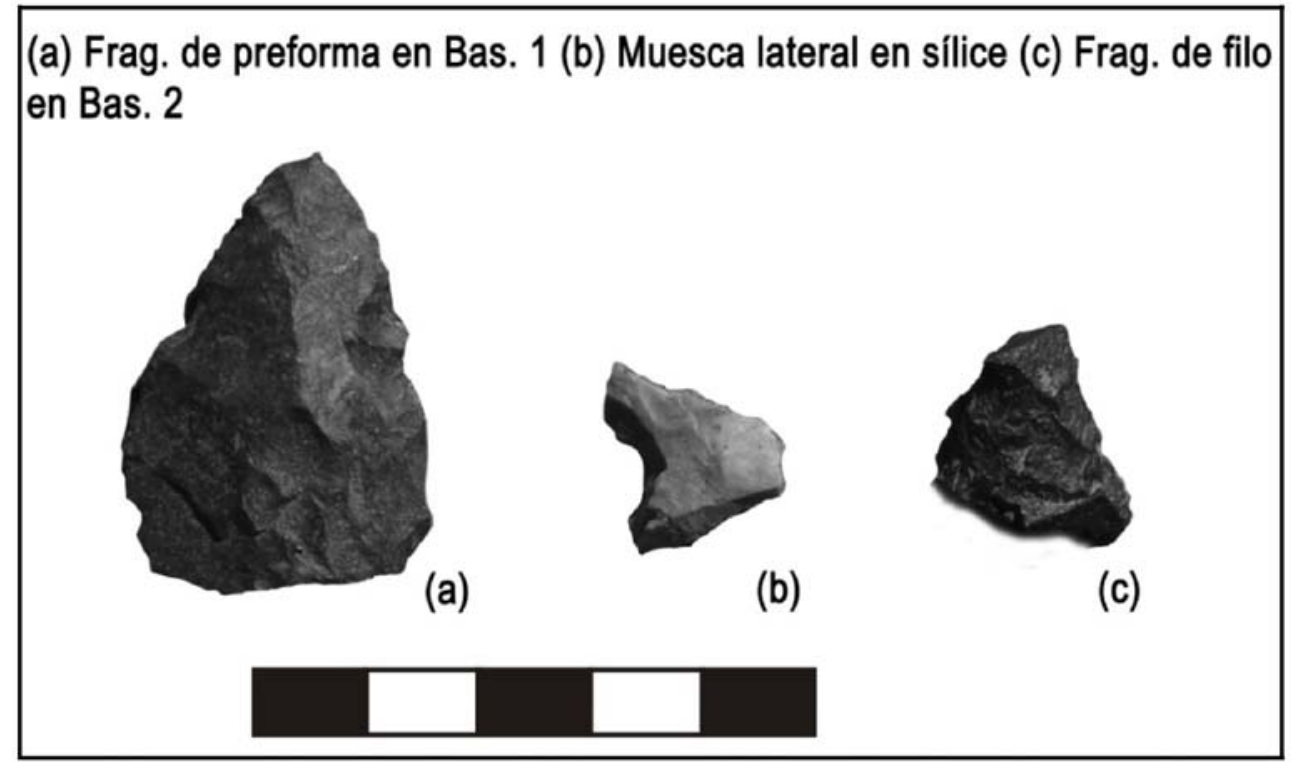

FIGURA 2: Tipos de instrumentos registrados en el conjunto 3 del sitio AMA 3.

En la TAB. 4, se puede observar el estado de descarte de los instrumentos por cada conjunto temporal. En el conjunto 2 hay sólo un instrumento embotado y entero, mientras que 5 aún están activos, de los cuales 3 están enteros. En el conjunto 3, los tres ins- trumentos se encuentran fragmentados, de los cuales uno esta activo. En el conjunto 4, los tres instrumentos se encuentran fragmentados, y uno de ellos embotado. 
TABELA 4: Estado de descarte de los instrumentos.

\begin{tabular}{|c|c|c|c|c|c|}
\hline \multirow{2}{*}{ Conjuntos } & \multirow{2}{*}{ Estado } & \multicolumn{3}{|c|}{ INSTRUMENTOS } & \multirow{2}{*}{ Total } \\
\cline { 3 - 5 } & & Activo & Embotado & Ind. & \\
\hline \multirow{2}{*}{$\mathbf{2}$} & Fragmentados & 2 & - & 1 & \multirow{2}{*}{7} \\
\cline { 2 - 5 } & enteros & 3 & 1 & - & \\
\hline \multirow{2}{*}{3} & Fragmentados & 1 & - & 2 & \multirow{2}{*}{3} \\
\cline { 2 - 5 } & enteros & - & - & - & \\
\hline \multirow{2}{*}{4} & Fragmentados & - & 1 & 2 & \multirow{2}{*}{3} \\
\cline { 2 - 5 } & enteros & - & - & - & \\
\hline
\end{tabular}

Aunque el conjunto 3 y 4 tienen la misma cantidad de instrumentos, cada conjunto tuvo una tasa de depositación diferente. Para llegar a interpretaciones más acertadas es necesario tener esto en consideración. Por ello, en la FIG. 3 se compara la tasa de depositación entre los tres conjuntos. La FIG. 3 muestra que el conjunto más temprano de ocupación (conjunto 4) y el más tardío (conjunto 2), tienen casi la misma tasa de depositación. Sin embargo, el conjunto 3, es el que presenta menor tasa de depositación. Sin embargo, en la TAB. 1, se puede observar que la duración cada conjunto es diferente. Por lo tanto, el conjunto 3, que es el que menos cantidad de instrumento tiene, es a su vez, el que más duración temporal tuvo. Es decir, en 2.360 años, sólo se depositaron 3 instrumentos. Mientras que en el conjunto 2, en menos cantidad de tiempo, se registró casi el doble de instrumentos. El conjunto 4, en comparación al conjunto 3 , en la mitad de tiempo se registró la misma cantidad de instrumentos. Entonces, teniendo en cuenta la TAB. 1 y la FIG. 3 , los conjuntos 2 y 4 son más parecidos en cuanto al registro de instrumentos hallados.

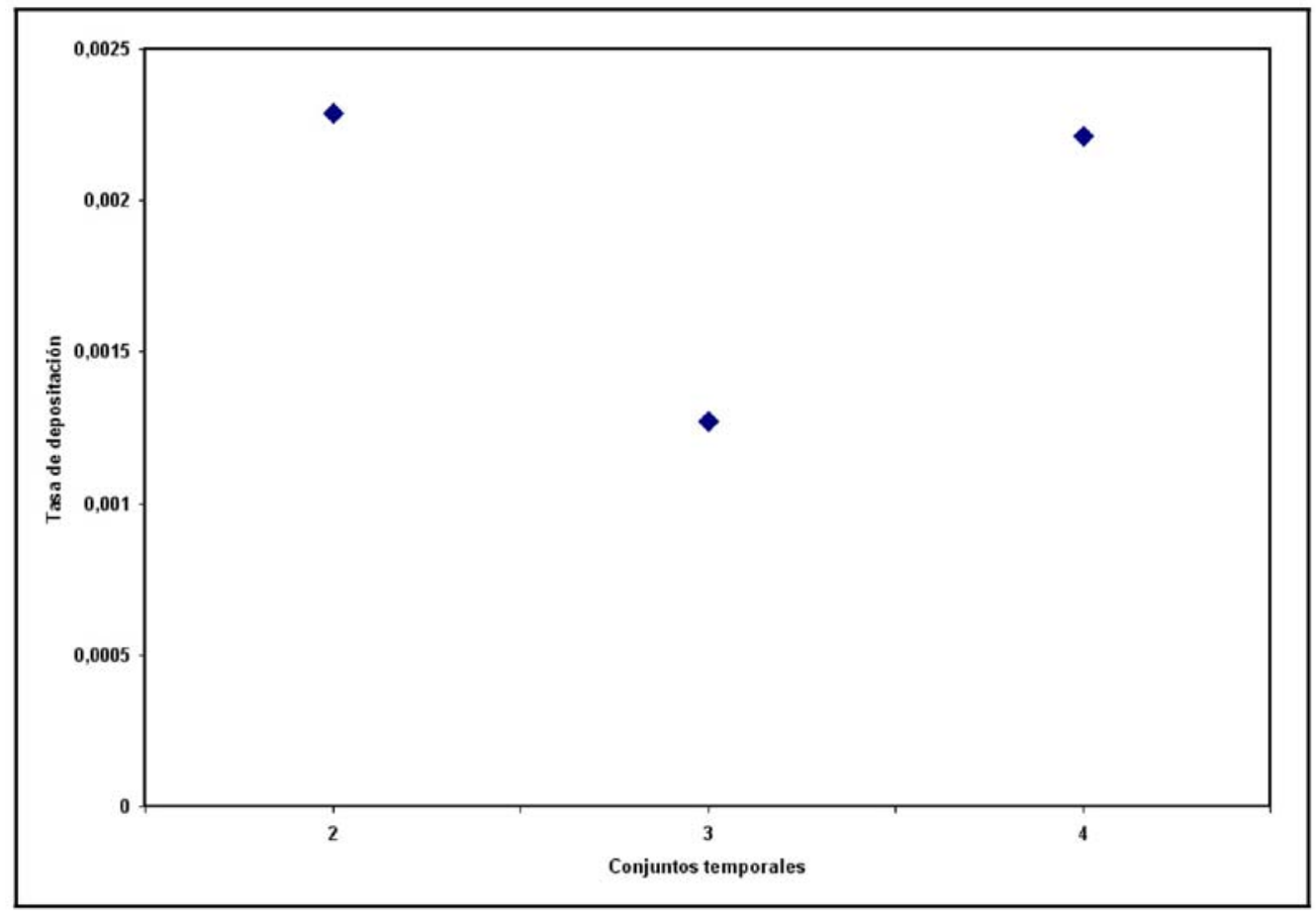

FIGURA3: Tasa de depositación de los instrumentos por conjuntos temporales. 


\section{DISCUSIÓN}

Los resultados obtenidos muestran algunas diferencias entre los tres conjuntos analizados. Las más marcadas se encuentran en el conjunto 3 (Holoceno medio) y están en relación con la frecuencia de las materias primas, sus tamaños, la cantidad de material depositado y los índices de fractura (PÉREZ WINTER, 2009a).

Como se mencionó en la introducción de este trabajo, durante el Holoceno medio se produjo un proceso de aridización. Gil et al. (2005) propusieron que esta aridez pudo producir una reducción de la biomasa y las fuentes de agua que estaban disponibles en el ambiente. Teniendo esto en consideración se discutirán las hipótesis propuestas.

Las dos hipótesis planteadas fueron: 1-Durante el Holoceno medio los grupos utilizaron una estrategia de maximización en la explotación de recursos. 2- Durante el Holoceno medio se dedicó más tiempo y energía a las actividades de subsistencia, dedicando menos tiempo y energía a las actividades tecnológicas.

Si bien el tamaño de la muestra dificulta realizar afirmaciones concluyentes a favor de una u otra hipótesis, algunas tendencias pueden orientarnos hacia alguna de ellas. Los datos obtenidos del análisis muestra que el conjunto 3 (Holoceno medio) es el que menos cantidad de instrumentos tiene. Los tres instrumentos registrados en este conjunto parecen ser extractivos. Estos se encuentran manufacturados en materias primas de muy buena y buena calidad para la talla. La energía puesta en la manufactura de esos instrumentos no parece ser muy costosa, salvo por la preforma. Los instrumentos se encontraron fracturados, y sólo uno en estado activo. Asimismo, en otra publicación se mostró que el conjunto 3 corresponde más a una estrategia conservada por la relación entre instrumentos / desechos y una estrategia de equipamiento personal de los individuos, que se corresponde a grupos con alta movilidad (PÉREZ WINTER, 2009a). Por lo tanto, teniendo en consi-deración las expectativas propuestas para cada hipótesis, la tendencia lítica del conjunto 3 parece acercarse más a la hipótesis número 1. Ya que los instrumentos están confeccionados en materias primas de buena calidad para la talla. La fractura de los instrumentos puede estar dado también por factores post - depositacionales (SHIFFER, 1987) que tendrán que ser evaluados en posteriores trabajos.

Sin embargo, si ampliamos la muestra y tenemos en consideración el análisis realizado por Garvey (2008) en Gruta del Manzano, correspondiente a la ocupación del Holoceno medio (TAB. 5), se observa que hay un mayor porcentaje de instrumentos informales. Estos son aquellos que tiene menor inversión de energía puestos en su manufactura (ANDREFSKY, 1994). De estos, más de la mitad están confeccionados en materias primas locales, que son de menor calidad que las no locales, como la obsidiana. La menor proporción esta dada por instrumentos formales, que son aquellos que llevan mayor inversión de energía en su manufactura (ANDREFSKY, 1994), confeccionados en obsidiana, al igual que los escasos desechos de reducción bifacial (GARVEY, 2008, p.7).

TABELA 5: Cantidad y porcentajes de los tipos de instrumento en Gruta el Manzano durante el Holoceno medio.

Extraído y modificado de Garvey, 2008.

\begin{tabular}{lcc}
\hline Líticos & cantidad & porcentaje \\
Materia prima local & 753 & $62 \%$ \\
Instrumentos informales & 97 & $61 \%$ \\
Instrumentos informales en material prima local & 62 & $64 \%$ \\
Materia prima no local & 456 & $38 \%$ \\
Instrumentos formales & 61 & $39 \%$ \\
Instrumentos formales en material prima no local & 19 & $31 \%$ \\
Reducción bifacial & 28 & $3 \%$ \\
Reducción bifacial en material prima no local & 18 & $64 \%$ \\
Todos los instrumentos & 158 & $13 \%$
\end{tabular}


Teniendo en cuenta los datos presentados, se podría decir que la hipótesis 2 es la más probable hasta el momento. Sin embargo, en el trabajo de Garvey (2008) no se especifican qué tipos de instrumentos están enmarcados dentro de los formales e informales, como para diferenciar entre instrumentos extractivos y mantenibles, y tampoco están publicados el estado de descarte de los instrumentos. Por lo tanto, teniendo en consideración las frecuencias, porcentajes y la materia prima utilizada, las tendencias líticas están apuntando más a la hipótesis 2. Sin embargo, se espera que futuros datos sean recolectados para testear el alcance de ambas hipótesis.

\section{CONSIDERACIONES FINALES}

El aporte de este trabajo es mostrar el potencial que tiene el análisis de los artefactos líticos a la hora de analizar las diferentes estrategias que los grupos humanos pudieron haber utilizado para confrontar cambios climáticos en el pasado. Asimismo, el análisis del material lítico ofrece datos con los cuales discutir temas como movilidad (KELLY, 1988), funcionalidad de sitio (BINFORD, 1998) y uso del espacio (FRANCO, 2002; CORTEGOSO, 2005; LUCERO et al. 2006). Por lo tanto, el aporte general de este trabajo es el empezar a enmarcar los análisis líticos en el sur de Mendoza, desde esta perspectiva. Es decir, analizar las estrategias y cambios en relación al contexto social y ambiental de los grupos humanos. Asimismo, se espera que con investigaciones futuras se pueda ampliar la muestra del registro lítico correspondiente al Holoceno medio para evaluar el alcance de las hipótesis propuestas.

Para finalizar, este trabajo abrió nuevos interrogantes que permiten continuar discutiendo ideas a nivel regional. Es necesario afinar ciertas cuestiones, como la realización de un diseño sistemático de localización de recursos lítico a escala regional (FRANCO y BORRERO, 1999). Esto permitirá, junto con muestras para realizar cortes delgados, una mayor precisión en la determinación de las fuentes de materias primas utilizadas y registradas en los sitios arqueológicos. De esta forma, con esos datos se podrán reconstruir los mínimos rango de acción e inferir, con argumentos más sólidos, aspectos sobre la organización tecnológica, uso diferencial de materias primas, movilidad, uso del espacio, entre otras cosas.

\section{AGRADECIMIENTOS}

Quiero agradecer a Agustín Martinelli por las correcciones y sugerencias realizadas a este trabajo. También a Adelino Carvalho por corregir el resumen en portugués.

\section{BIBLIOGRAFÍA}

ABRAHAM, E. M., GARLEFF, K., LIEBRICHT, H., REGAIRAZ, A., SCHÄBITZ, F., SQUEO, F., STINGL, H., VEIT H. Y VILLAGRÁN C. Geomorphology and Paleoecology of the Arid Diagonal in Souther South America. En: Zietchrift für Angewandte Geologie, Hannover: 55-62. Ed. Miller H. y Hervé F., Alemania. 2000.

ANDREFSKY, W. Raw material availability and the organization of the technology. American Antiquity 54 (1): 166-168. 1994.

ARAUJO, A., NEVES, W., PILÓ, L. Y ATUI, J. Holocene dryness and human occupation in Brazil during the "Archaic Gap". Quaternary Research, 64: 298-307. 2005.

BAMFORTH, D. B. y BLEED, P. Technology, flaked stone technology, and risk. En: Rediscovering Darwin: Evolutionary Theory and Archeological: 109-139. Ed. Barton, C. M. y Clark, G. A. American Anthropological Association, Washington. 1997.

BINFORD, L. Interassemblage variability - the Mousterian and the 'functional' argument. En: The explanation of culture change: models in Prehistory: 227-254. Ed. Renfrew, C.. Duckworth, London. 1973.

Organization and formation processes: looking at curated technologies. Journal of Anthropological Research, 35: 255-273. 1979.

En busca del Pasado. Ed. Crítica (reinpresión de la primera edición 1988), Barcelona. 1998. 
BOUSMAN, C. B. Hunter - gatherer adaptions, economic risk and tool design. Lithic Technology 18 (1 y 2): 59-86. 1993.

Coping with risk: Later Stone Age technological strategies at Blydefontein Rock Shelter, South Africa. Journal of Anthropological Archaeology 24: 193-226 2005.

BRACCO, R., DEL PUERTO, L., INDA, H. Y CASTIÑEIRA, C. Mid-late Holocene cultural and environmental dynamics in Eastern Uruguay. Quaternary International 132: 37-45. 2005.

BRYSON, R. On integrating climatic change and culture change study. Human Ecology 22 (1):115-128. 1994.

CASHDAN, E. Spatial organization and habitat use. En: Evolutionary Ecology and Human Behavior:237266. Ed. E. Smith y B. Winterhalder. Aldine de Gruyter. New York. 1992.

CARR, P. J. Technological Organization and Prehistoric Hunter-Gatherer Mobility: Examination of the Hayes Sites. En: The organization of North American prehistoric chipped stone Technologies: 35-44. Ed. Carr, P. J. Internacional Monographs in Prehistory. Archaeological Series 7. Michigan. 1994.

CORTEGOSO, V. Mid Holocene hunters in the Andes Mountains: environment, resources and technological strategies. Quaternary International,132: 71-80. 2005.

D’ANTONI, H. Los últimos treinta mil años en el sur de Mendoza. Memorias del III coloquio sobre paleobotánica y palinología: 83-103. INAH. 1980.

DIEGUEZ, S., Y NEME, G. Geochronology of the archaeological site Arroyo Malo 3 and the first human occupations in the Northpatagonia early Holocene. En: Ancient Evidence for Paleo South Americans: From Where the South Winds Blows: 87-92. Ed. Miotti, L.; Salemme M., y Flegenheimer, N. University Press, Texas. 2003.
DINCAUZE, D. Environmental Archaeology. Principal and practice. Cambridge Press, London. 2000.

DURÁN, V. Poblaciones Indígenas de Malargüe, su arqueología e historia. Universidad Nacional de Cuyo/ Facultad de Filosofía y Letras. CEIDER, Serie Libros $\mathrm{N}^{\circ}$ 1. 2004.

FRANCO, V. N. ¿ Es posible diferenciar los conjuntos líticos atribuidos a la exploración de un espacio de los correspondientes a otras etapas del poblamiento? Werken 3: 119-132. 2002.

La organización tecnológica y el uso de las escalas espaciales amplias. El caso del sur y oeste de Lago Argentino. Temas de Arqueología, Análisis Lítico: 101-144. Ed. Acosta, A., y Loponte, D. y M. Ramos, Buenos Aires. 2004.

FRANCO, V. N. Y BORRERO, L. A. Metodología de análisis de la estructura regional de recursos líticos. En: En los tres reinos de recolección. Cono Sur de América: 27-37. Ed. Aschero, C., Kostanje, A. y Vuoto, C. Instituto de Arqueología y Museo, Tucumán. 1999.

GAMBLE, C. Y SOFFER, O. Introduction. Pleistoce polyphony: the diversity of human adaptations at the Last Glacial Maximum. En: The World at 18,000 B.P. 1: 1-23. Eds. Soffer, O. y Gamble, C.. Unwin Hyman, London. 1990.

GARVEY, R. A behavioral ecological approach to a proposed middle Holocene occupational gap. Before Farming 2 (2): 1-14. 2008

GIL, A. Arqueología de la Payunia (Mendoza, Argentina). El poblamiento humano en los márgenes de la agricultura. Ed. BAR Series 1477, Inglaterra. 2006.

GIL, A., ZÁRATE, M. Y NEME, G. Mid-Holocene Paleoenvironments and the archaeological record of southern Mendoza, Argentina. Quaternary International 132: 81-94. 2005.

GONZALES DÍAZ, E. y FAUQUÉ, L. Geomorfología. XII Congreso Geológico Argentino y II Congreso de 
Exploración de Hidrocarburos. Geología y Recursos Naturales de Mendoza, Mendoza: 217-224. 1993.

HALSTEAD, P. The economy has a normal surplus: ecnomic stability and social change among early farming communities of Thessaly, Greece. En: Bad Year Economics: 68 80. Ed. Hastled, P. y O’Shea, J. Cambridge University Press. 1989.

HALSTEAD, P. Y O'SHEA, J. Introduction: cultural responses to risk and uncertainty. En: Bad Year Economics: 1 7. Ed. Hastled, P. y O'Shea, J.. Cambridge University Press. 1989.

JESKE, R. J. Energetic Efficiency and Lithic Technology: An Upper Mississippian Example. American Antiquity 57 (3): 467-481. 1992.

JONES, T. L., BROWN, G. M., RAAB, L. M., MCVICKAR, J. L., SPAULDING, W. G., KENNETT, YORK D. J., Y WALKER, P. L. Environmental imperatives reconsidered. Demographic crises in Western North America during the Medieval Climatic Anomaly. Current Anthropology 40: 137-170. 1999.

KELLY, R. L. The foraging spectrum. Diversity in hunter-gatherer lifeway. Simthsonian Institution Press. Washington D. C. 1995.

LAGIGLIA, H. 1997a. Arqueología prehistórica del Atuel y del Diamante. Revista del CINTER, 2: 29-46.

Arqueología de cazadores-recolectores de altura. Norte del volcán Overo, El sosneado, San RafaelMendoza. Ediciones Ciencia y Arte. San Rafael. 1997b.

El Holoceno medio en el Atuel. En: Simposio La Ocupación Humana en el Holoceno medio: 253. XVI Congreso Nacional de Arqueología Argentina, Rosario, Argentina. 2001.

Arqueología prehistórica del sur mendocino y sus relaciones con el centro oeste argentino. En: Entre Montañas y Desiertos. Arqueología del Sur de Mendoza: 43-65. Ed. Gil, A. y Neme, G. Sociedad Argentina de Antropología; Buenos Aires. 2002.
Exploraciones arqueológicas y ambientales en los altos valles de las leñas y aledaños. III Taller Binacional argentino-chileno "Arqueología de la Cordillera de Los Andes 32\% $/ 40^{\circ}$ Latitud Sur. Museo Municipal de Historia Natural Notas del Museo 58: 37-40. Departamento de San Rafael, Provincia de Mendoza. 2005.

LLANO, C. Recursos Vegetales y Ocupaciones Humanas: Perspectivas Arqueobotánicas en el Sur de Mendoza. Tesis de Licenciatura. Universidad del Aconcagua, Mendoza. 2005.

LUCERO, G., CORTEGOSO V. Y CASTRO S. Cazadores-recolectores del holoceno Temprano: Explotación de Recursos líticos en el Sitio Agua de la cueva Sector Norte. En: Arqueología y Ambiente de Áreas Naturales Protegidas de la Provincia de Mendoza: 185-215. Ed: Durán V. y Cortegoso, V., Mendoza. 2006.

MANCINI, M. V., PAEZ, M. M:, PRIETO, A. R., STUTZ, S., TONILLO, M Y VILANOVA, I. MidHolocene climatic variability reconstruction from pollen records (32 ${ }^{\circ}-52^{\circ} \mathrm{S}$, Argentina). Quaternary International,132: 47-59. 2005.

MARKGRAF, V. Late posglacial vegetacional and paleoclimatic change in subartic temperature and arid environments in Argentine. Palynology 7: 43-70.1983.

MELTZER, D. Human Responses to Middel Holocene (Altithermal) Climates on the North American Great Plains. Quaternary Research 52: 404-416. 1999.

NELSON, M. 1991. The Study of Technological Organization. Archaeological Method and Theory: 5799. Ed. Schiffer, M. Tucson University of Arizona Press.

Technological Strategies Responsive to Subsistence Stress. En: Evolving Complexity and Environment Risk in the Prehistory of Southwest: 107143. Ed. Tainter, J. y Tainter, B. Addison-Wesley. 1995.

NEME, G. Cazadores-recolectores de altura en los 
Andes meridionales: el alto valle del río Atuel. British Archaeological Reports Iternational. BAR Series: 1591. 2007.

NEME, G., MOREIRA, G., ATENCIO, G. Y DESANTE, A. El registro de microvertebrados del sitio arqueológico Arroyo Malo 3 (Provincia de Mendoza; Argentina). Revista Chilena de Historia Natural, 75: 410-513. 2002.

NORTE, F. Mapa climático de Mendoza. En: Recursos y problemas ambientales de zona árida. Primera parte: provincias de Mendoza, San Juan y La Rioja. Tomo I: Caracterización ambiental: 25-27. IDIZA. Ed. Abraham M. E. y Rodriguez Maritnez, F. Mendoza. 2000.

NUÑEZ, L., GROSJEAN, M.Y CARTAGENA I. Human Dimensions of Late Pleistocene/Holocene Arid Events in Souther South America. En Interhemisferic Climate Linkages: 105-117. Ed. Markgraf., V. New York Academic Press. 2002.

O'SHEA, J. M. The role of wild resources in smallscale agricultural systems: tales from the Lakes and the Plains. En: Bad Year Economics: 57 67. Ed. Hastled, P. y O'Shea, J. Cambridge University Press. 1989.

PARUELO, L. M., JOBBÁGY, E. G. Y SALA, O. E. Current Distribution of Ecosystem Functional Types in Temperate South America. Ecosystems 4: 683-698. 2001.

PÉREZ WINTER, C. Tendencias en el registro lítico durante el Holoceno medio: sitio Arroyo malo 3, alto valle del río Atuel, sur de Mendoza. Intersecciones (Enprensa). 2009a.

Estructuración de los recursos líticos en la provincial del sur de Mendoza, Argentina: una perspectiva arqueológica. Caminhos de geografía 9 (29): 1-12. 2009b.

PROHASKA, F. The Climate of Argentina, Paraguay and Uruguay. En: Climates of Central and South
America: 13-112. Ed. Scheraltfeger y Elsevier W., Ámsterdam. 1976.

SCHIFFER, M. B. Formation Processes of the Archaeological Record. Albuquerque: University of New Mexico Press. 1987.

SQUEO, P., CEPEDA, P., OLIVARES, N. Y ARROYO, M. Interacciones ecológicas en la alta montaña del valle del Elqui. Geología de los Andes desérticos. En: La Alta montaña del Valle del Elqui: 69-103. Ed. Cepeda, J. Ediciones Universidad de La Serena, Chile. 2006.

STINGL, H. Y GARLEF, K. Glacier variations and climate of the late quaternary in the subtropical and mid - latitude andes of Argentina. Zeitschrisft für Gletscherkunde und Glazialgeologie 21: 225-228. 1995.

TORRENCE, R. Retooling: towards a behavioral theory of stone tools. En: Time, energy and stone tools: 57-66. Ed. Torrance, R. Cambridge University Press. 1989.

VOLKHEIMER, W. Descripción geológica de la Hoja 27b, cerro Sonseado, provincia de Mendoza. Ministerio de Economía, Secretaría de Estado de Minería. Servicio Geológico Nacional, Buenos Aires. 1978.

WIESSNER, P. Risk, reciprocity and social influences on !Kung San economies. En: Politics and history in band societies: 61-84. Ed. Leacock, L. y Lee, R. Cambridge University Press, Cambridge. 1982.

WINTERHALDER, B. Y GOLAND, C. An evolutionary ecology perspective on diet choice, risk, and plant domestication. En: Peoples, Plants and Landscapes Studies in Paleoethnobotany: 123-160. Ed. Gremillion, K. University of Alabama Press. 1997.

YACOBACCIO, H. Sociedad y ambiente en el NOA precolombino. En: El hombre y su tierra: 26- 38. Buenos Aires. 1996.

YACOBACCIO, H. Y MORALES, M. Mid-Holocene 
environment and human occupation of the Puna (Susques, Argentina). International Quaternary, 132: 5- 14. 2005.

ZÁRATE, M. Los ambientes del tardiglacial y holoceno en Mendoza. En: Entre Montañas y Desiertos. Arqueología del Sur de Mendoza: 9-42. Ed. Gil, A. y Neme, G. Sociedad Argentina de Antropología. Buenos Aires. 2002. 